\title{
Análise do Senso de Coerência, ansiedade e depressão em alunos de pós-graduação: Estudo follow up
}

Analysis of the Sense of Coherence, anxiety and depression in graduate students: Follow-up study Análisis del sentido de Coherencia, ansiedade y depresión em estudiantes graduados: Estudio de seguimento

Recebido: 24/05/2021 | Revisado: 04/06/2021 | Aceito: 08/06/2021 | Publicado: 21/06/2021

Priscila Florentino Silva

ORCID: https://orcid.org/0000-0002-9370-4335 Universidade de Pernambuco, Brasil E-mail: priscilafsilva2007@yahoo.com.br

Arnaldo de França Caldas Júnior ORCID: https://orcid.org/0000-0002-3713-7532 Universidade de Pernambuco, Brasil E-mail: caldasjr@alldeia.com.br

Diana Santana de Albuquerque ORCID: https://orcid.org/0000-0002-7897-2489 Universidade de Pernambuco, Brasil E-mail: diana.albuquerque@upe.br

\begin{abstract}
Resumo
O interesse pela pesquisa que afere a ansiedade, depressão e resiliência entre estudantes de pós-graduação da área de saúde durante o curso tem aumentado nos últimos anos. Este estudo analítico follow-up avaliou a presença de ansiedade, depressão e senso de coerência (resiliência) em estudantes de pós-graduação da UPE e UFPB de Odontologia e outros cursos (Enfermagem, Educação Física, Hebiatria, Perícias Forenses). Foi utilizado um questionário socioeconômico, seguido do questionário de Senso de Coerência (SOC) que mensura a capacidade do indivíduo em ser adaptativo aos eventos diários. O Inventário de Ansiedade Estado-Traço (STAI) mediu a ansiedadeestado (sobre um evento) e ansiedade-traço (característica pessoal). O Inventário de Depressão de Beck (BDI-II) foi o quarto instrumento dessa pesquisa. A primeira coleta de dados foi realizada nas aulas inaugurais das turmas de mestrado 2019/2020 e a segunda coleta de dados foi realizada no momento da qualificação dos alunos. Os participantes da pesquisa apresentaram aumento no valor do senso de coerência, aumento nos níveis de ansiedade-estado de $14,6 \%$ para $36,7 \%$ ( $\mathrm{p}<0.001)$ e a ansiedade-traço continuou com níveis altos. No segundo momento 2 alunos apresentaram depressão severa e 72,5\% mínima depressão. Conclui-se que houve um aumento do senso de coerência, surgimento da depressão severa, ansiedade-traço no nível moderado e o aumento da ansiedade-estado ( $\mathrm{p}<0,001)$ entre os alunos pósgraduandos pesquisados.
\end{abstract}

Palavras-chave: Ansiedade; Depressão; Resiliência psicológica.

\begin{abstract}
Interest in research that measures anxiety, depression and resilience among graduate students in the health area during the course has increased in the last years. This follow-up analytical study evaluated the presence of anxiety, depression and a sense of coherence (resilience) in graduate students at UPE and UFPB in Dentistry and other courses (Nursing, Physical Education, Hebiatry, Forensic Skills). A socio-economic questionnaire was used, followed by the Sense of Coherence questionnaire (SOC) that measures the individual's ability to be adaptive to daily events. The State-Trait Anxiety Inventory (STAI) measured state-anxiety (about an event) and trait-anxiety (personal characteristic). The Beck Depression Inventory (BDI-II) was the fourth instrument in this research. The first data collection was performed in the inaugural classes of the 2019/2020 master classes, the second data collection was performed at the time of the students' qualification. Research participants showed an increase in the sense of coherence, an increase in anxiety-state levels from $14.6 \%$ to $36.7 \%$ ( $\mathrm{p}<0.001$ ) and trait-anxiety continued with high levels. In the second moment, 2 students presented severe depression and $72.5 \%$ minimal depression. It was concluded that there was an increase in the sense of coherence, the emergence of severe depression, trace-anxiety at a moderate level and an increase in state-anxiety ( $p$ $<0.001)$ among the graduate students surveyed.
\end{abstract}

Keywords: Anxiety; Depression; Resilience psychological.

\section{Resumen}

El interés por la investigación que evalúe la ansiedad, la depresión y la resiliencia entre los estudiantes de posgrado en el área de la salud durante el curso ha aumentado en los últimos años. Este estudio de seguimiento analítico evaluó la 
presencia de ansiedad, depresión y sentido de coherencia (resiliencia) en estudiantes de posgrado de la UPE y UFPB en Odontología y otros cursos (Enfermería, Educación Física, Hebiatria, Habilidades Forenses). Se utilizó un cuestionario socioeconómico, seguido del cuestionario de sentido de coherencia (SOC) que mide la capacidad del individuo para adaptarse a los eventos diarios. El Inventario de Ansiedad Estado-Rasgo (STAI) midió el estado-ansiedad (acerca de un evento) y el rasgo de ansiedad (característica personal). El Inventario de Depresión de Beck (BDI-II) fue el cuarto instrumento de esta investigación. La primera recolección de datos se realizó en las clases inaugurales de las clases magistrales (2019/2020) y la segunda recolección de datos se realizó en el momento de la calificación de los estudiantes. Los participantes de la investigación mostraron un aumento en el sentido de coherencia, un aumento en los niveles de estado de ansiedad del 14,6\% al 36,7\% (p <0,001) y la ansiedad rasgo continuó con niveles altos. En el segundo momento, 2 estudiantes presentaron depresión severa y 72,5\% depresión mínima. Se concluyó que hubo un aumento en el sentido de coherencia, la aparición de depresión severa, traza de ansiedad en un nivel moderado y un aumento en el estado de ansiedad ( $p<0,001)$ entre los estudiantes de posgrado encuestados.

Palabras clave: Ansiedad; Depresión; Resiliencia psicológica.

\section{Introdução}

A proporção da população global com depressão e ansiedade em 2015 era estimada em 4,4\% e 3,6\% sendo a depressão e a ansiedade mais comuns entre mulheres (5,1 e 4,6\%) do que em homens $(3,6$ e 2,6\%) (World Health Organization, 2017). Segundo dados de 2020, a OMS afirmou que globalmente mais de 264 milhões de pessoas de todas as idades sofrem de depressão e mais mulheres são afetadas do que homens. A depressão é um transtorno mental comum sendo uma das principais causas de deficiência em todo o mundo e é um dos principais contribuintes para o desenvolvimento de doenças (World Health Organization, 2020).

A depressão é um distúrbio de humor que causa sintomas angustiantes que afetam a maneira como você sente, pensa e lida com as atividades diárias, como dormir, comer ou trabalhar. Para ser diagnosticado com depressão, os sintomas devem estar presentes a maior parte do dia, quase todos os dias por pelo menos 2 semanas (APA, 2014). Os transtornos de ansiedade são sofrimentos subjetivos devido à preocupação constante e podem interferir nas atividades diárias, como o trabalho, escola e relacionamentos. As pessoas com transtorno de ansiedade generalizada (TAG) demonstram ansiedade ou preocupação excessiva, na maioria dos dias por pelo menos 6 meses, sobre várias coisas, como saúde pessoal, trabalho, interações sociais e circunstâncias da vida cotidiana. (APA, 2014).

Há uma crescente literatura que analisa a prevalência e os fatores causais de ansiedade e depressão durante o período de graduação e pós-graduação nas universidades (Zhang et al., 2015; Puthran et al., 2016) como a maior exigência de produção científica internacional (Cirani \& Campanario, 2015; Iriart \& Trad, 2020), a insegurança criada pela incerteza da oferta de bolsas e no que se refere ao futuro do recém-formado, no contexto atual da instabilidade política nacional e mundial (Costa, 2019). Esses fatores podem se apresentar como estressores no dia a dia dos pós-graduandos, prejudicando os seus desempenhos, desestimulando-os ou produzindo distúrbios como ansiedade e depressão (Wanderley, 2017). Os transtornos de ansiedade e depressão são as principais categorias diagnósticas de distúrbios psicológicos e têm alta prevalência no mundo, entre os estudantes é crescente e diminui a capacidade de sua performance na sala de aula e fora dela e na interação social com familiares e amigos (Woolston, 2019).

Por outro lado, com os desafios existentes na pós-graduação, os estudantes podem desenvolver um senso de autonomia diante dos problemas, bom relacionamento com os colegas de turma e otimizar a tomada de decisão em situações adversas, reforçando sua capacidade adaptativa às situações diárias (resiliência) (Klawonn et al., 2019; Jeong \&, Koh, 2021). A abordagem salutogênica (promoção da saúde) nos currículos de educação em saúde é bem-sucedida e a pesquisa no tema é importante para identificar fatores de promoção da saúde durante programas de educação (Klawonn et al., 2019; Lindmark et al., 2020). Tem-se evidenciado que pessoas com um alto Senso de Coerência apresentam (SOC) uma boa percepção de sua saúde, melhor qualidade de vida e é considerado hoje na área da saúde como uma nova abordagem para a avaliação de indivíduos em condições crônicas de saúde ou pertencentes a grupos específicos, como idosos, estudantes, adolescentes, 
gestantes e crianças (Greimel et al., 2016). Indivíduos com um alto SOC avaliam situações como não estressantes (compreensibilidade), percebem recursos a serem prontamente disponíveis para lidar com o estresse (capacidade de gerenciamento), e veem os eventos da vida e a doença como desafios dignos de esforço (significância) (Antonovsky, 1993; Lindström \& Eriksson, 2011).

Assim, o presente estudo objetivou verificar a presença de resiliência, ansiedade e depressão em estudantes de pósgraduação de duas universidades Nordestinas: Universidade de Pernambuco (UPE) e Universidade Federal da Paraíba (UFPB) através do índice Senso de Coerência (Antonovsky, 1993) (SOC), do Inventário de Ansiedade Estado-Traço (Spielberger et al., 1983) (STAI) e do Beck Depression Inventory (Beck, 1961) em diferentes momentos do calendário acadêmico.

\section{Metodologia}

Este foi um estudo analítico de follow up, onde foram coletadas informações acerca da resiliência, ansiedade e depressão dos estudantes de pós-graduação em dois momentos do curso (aula inaugural e anteriormente à qualificação). Os instrumentos utilizados para a obtenção das informações relativas à ansiedade, senso de coerência e depressão dos pósgraduandos, nos dois momentos de coleta, foram os questionários: Senso de Coerência (SOC), Inventário de Ansiedade Estado-Traço (Stait Trait Anxiety Inventory-STAI) e Beck Depression Inventory - II (BDI-II).

\section{Amostra}

O projeto de pesquisa foi aprovado no Comitê de Ética em Pesquisa através do parecer 03699618.8.0000.5207. Foi solicitado autorização prévia à reitoria e à coordenação dos cursos de pós-graduação das universidades onde o estudo foi desenvolvido e, durante a coleta de dados, assinatura pelos participantes da pesquisa do Termo de Consentimento Livre e Esclarecido, sendo garantido o anonimato e o direito de desistência em qualquer fase da pesquisa. Obtivemos a informação do quantitativo de alunos selecionados, matriculados e contatos através das listas fornecidas pelas secretarias de pós-graduação dos cursos analisados. Dos 140 alunos matriculados e contactados obteve-se resposta inicial de 123 alunos no momento da aula inaugural, assim, totalizando nossa amostra final da primeira coleta. Foram excluídos da amostra mestrandos que estivessem utilizando medicamentos para depressão ou ansiedade. A amostra foi composta por alunos de pós-graduandos matriculados nos cursos de odontologia, enfermagem, educação física (UPE e UFPB), perícias forenses e hebiatria (UPE) no biênio 2019/2020 (UPE, 2021; UFPB, 2021).

A primeira coleta de dados foi realizada nas aulas inaugurais das turmas de mestrado (2019) onde, nesse momento, a pesquisadora explicou os objetivos da pesquisa aos participantes, o sigilo das informações coletadas e a liberdade de desistência da participação dos estudantes. A segunda coleta de dados foi realizada durante o período de qualificação do projeto de pesquisa de cada aluno por meio do Google Forms onde 98 (79\%) dos pesquisados retornaram o questionário respondido.

\section{Instrumentos de coleta}

O instrumento de coleta de dados foi organizado em quatro etapas contínuas, cada uma constituída de questões objetivas, sendo aplicado aos alunos de pós-graduação devidamente matriculados nas Universidades citadas, para levantamento de aspectos distintos, conforme descrito a seguir. 


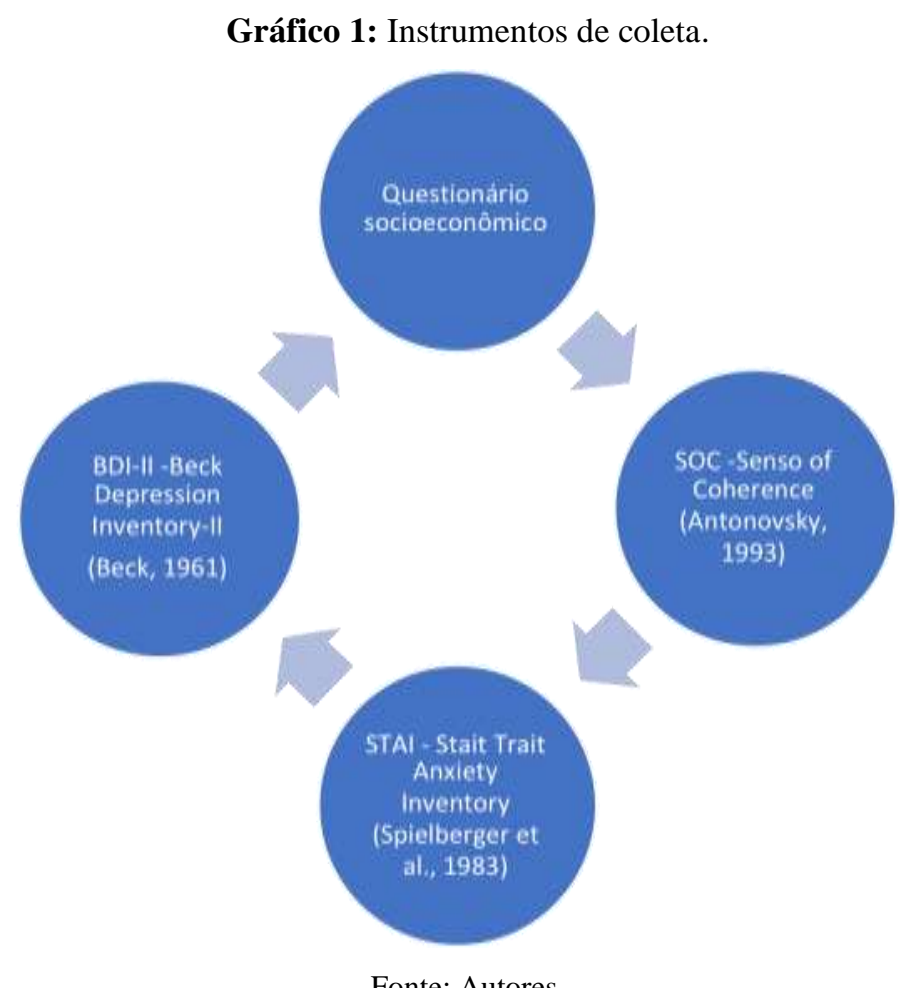

a) questionário socioeconômico: com objetivo de levantar dados e hábitos dos participantes, permitindo a caracterização do aluno da sua condição familiar, estudantil e social;

b) o SOC (Senso de Coerência) [(Antonovsky, 1993)]: com o objetivo de mensurar a capacidade de enfrentamento de situações estressantes no dia a dia estudantil, composto por 13 questões na sua versão adaptada trans culturalmente por Freire, Sheiham, e Hardy (2001) onde as respostas estão em uma escala tipo Likert de 5 pontos;

c) o STAI (Inventário de ansiedade estado-traço): com objetivo de identificar, medir e caracterizar o tipo de ansiedade baseado em uma escala Likert de 4 pontos e consiste em 40 breves perguntas de autorrelato e mede dois tipos de ansiedade: ansiedade-estado (sobre um evento) e ansiedade-traço (característica pessoal) [(Spielberger et al., 1983)].

d) o BDI-II (Beck Depression Inventory): com objetivo de identificar a depressão, se presente, e medir a sua gravidade. Criado por Aaron T. Beck (1961), é um inventário de autorrelato de múltipla escolha com 21 perguntas, cada uma sendo pontuada em um valor de escala de 0 a 3.

\section{Análise de dados}

Inicialmente os dados foram consolidados, as análises realizadas no software SPSS (Statistical Package for the Social Sciences) versão 13 e os resultados das variáveis categóricas foram apresentados em forma de tabelas com suas respectivas frequências absolutas e relativas. Foi utilizado o teste de Qui-quadrado de Pearson para verificar possíveis associações entre as variáveis categóricas. Foi utilizado o teste de Normalidade de Kolmogorov-Smirnov para variáveis quantitativas.

Para verificar se existiam diferenças estatisticamente significativas entre variáveis numéricas e categóricas, foi utilizado o teste t Student (Distribuição Normal) ou teste não paramétrico de Mann-Whitney (Distribuição Não Normal) para grupos de duas categorias. O teste de ANOVA (Distribuição Normal) e/ou teste de Kruskal-Wallis (Distribuição Não Normal) foi utilizado para grupos com mais de duas categorias. 


\section{Resultados}

\section{Primeira coleta de dados}

Participaram da primeira coleta de dados 123 estudantes de pós-graduação (Tabela 1), cujo perfil em relação ao gênero foi de $65 \%$ feminino e $35 \%$ masculino. O curso de pós-graduação em Educação Física (convênio entre as duas Universidades: UPE e UFPB, constituindo uma única turma), apresentou o maior número de alunos (19,5\%) seguido pelo de Odontologia na UFPB $(16,3 \%)$.

A maioria dos estudantes pesquisados eram solteiros $(77,2 \%)$, sem filhos $(77,2 \%)$, com renda familiar de até 3 salários-mínimos $(17,1 \%)$ e a faixa etária nesse estudo variou de 21 a 69 anos com 70,4\% dos estudantes entre 20-30 anos. Trabalhar ao mesmo tempo em que cursa a pós-graduação foi relatado por 58,5\% dos estudantes. Morar na mesma cidade em que cursa a pós-graduação foi relatado por 57,7\% e 65,9\% já cursou alguma pós-graduação anteriormente. Quanto ao desempenho acadêmico $84,6 \%$ dos estudantes afirmam estar satisfeito com o desempenho acadêmico e $87 \%$ não sente dificuldades de aprendizagem. Seguir alguma religião foi relatado por 87,8\% dos estudantes e 17,9\% têm problemas de sono. Relativo as atividades físicas $26,8 \%$ relataram fazer 3 vezes na semana e $37,4 \%$ dos pesquisados relataram não fazer exercícios. 
Tabela 1: Distribuição dos estudantes de pós-graduação segundo as variáveis sexo, curso, faixa etária, religião, estado civil, renda mensal e número de filhos.

\section{VARIÁVEIS}

\section{Gênero}

Feminino

Masculino

Curso

Odontologia UPE

Perícias Forenses UPE 14

Enfermagem UPE 18

Hebiatria UPE 18

Odontologia UFPB 20

Enfermagem UFPB 14

Educação Física UPE/UFPB 24

Total

Estado civil

Solteiro

Casado

união estável

Separado

Outros

\section{Possui filhos}

Não $\quad 95 \quad 77,2$

Sim

Renda familiar

1 salário-mínimo

$2 \mathrm{SM}$

$3 \mathrm{SM}$

$4 \mathrm{SM}$

$5 \mathrm{SM}$

6-10 SM

Acima de 10 SM

não responderam

Idade

20-30

$31-40$

41-50

50 ou +
123

28

$N$

80

43

15

14

8

8

(1)

4

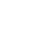

80

30

10

1

2

9
11
21
8
13
15
20
18

18

86

30

3

3
7,3

8,9

17,1

6,5

10,6

12

16

14,4

70,4

24,5

2,4

2,4

\section{VARIÁVEIS}

Trabalha

Sim

N $\quad \%$

Não

$72 \quad 58,5$

Mora na cidade em que faz a pós

Sim

$71 \quad 57,7$

Não

Já é pós-graduado

Sim

81

65,9

Não

42

Sente dificuldades de aprendizagem

Sim

15

12,2

100

Não

107

87

Tem problemas para dormir

Sim

22

17,9

Não

101

82,1

Segue alguma religião

Sim

$108 \quad 87,8$

Não

15

12,2

Faz atividades físicas com que frequência

Nenhuma

2 vezes por semana

3 vezes por semana

4 vezes por semana

5 vezes por semana

6 vezes por semana

todos os dias

sem frequência regular

não responderam

Satisfeito com o desempenho acadêmico

muito satisfeito

14

Satisfeito

104

Insatisfeito

muito insatisfeito

4

1
37,4

7,3

26,8

5,7

9,8

3,3

7,3

1,6

0,8

11,4

84,6

3,3

0,8

Fonte: Autores.

Na primeira coleta de dados houve a associação entre os índices de baixa resiliência (SOC) e moderada ansiedadetraço (STAI-2), e da associação de valores dos índices de moderada ansiedade-traço (STAI-2) com depressão mínima, medido pelo BDI-II conforme demonstrado na Tabela 2. 
Tabela 2: Correlação entre os índices STAI-2 e SOC e entre STAI-2 e BDI-II em estudantes de pós-graduação UPE e UFPB na primeira coleta de dados.

\begin{tabular}{|c|c|c|c|c|c|c|c|}
\hline \multicolumn{8}{|c|}{ SOC } \\
\hline & & & SOC Baixo & SOC Alto & TOTAL & p-valor & \\
\hline \multirow[t]{9}{*}{ STAI 2} & Baixo & Contagem & 8 & 13 & 21 & & \\
\hline & & $\%$ em STAI 2 & $38,1 \%$ & $61,9 \%$ & $100,0 \%$ & & \\
\hline & & $\%$ em SOC & $13,1 \%$ & $31,7 \%$ & $20,6 \%$ & & \\
\hline & Moderado & Contagem & 36 & 27 & 63 & & \\
\hline & & \% em STAI_2 & $57,1 \%$ & $42,9 \%$ & $100,0 \%$ & $=0,001 *$ & \\
\hline & & $\%$ em SOC & $59,0 \%$ & $65,9 \%$ & $61,8 \%$ & & \\
\hline & Alto & Contagem & 17 & 1 & 18 & & \\
\hline & & $\%$ em STAI 2 & $94,4 \%$ & $5,6 \%$ & $100,0 \%$ & & \\
\hline & & $\%$ em SOC & $27,9 \%$ & $2,4 \%$ & $17,6 \%$ & & \\
\hline \multirow[t]{5}{*}{ TOTAL } & & Contagem & 61 & 41 & 102 & & \\
\hline & & $\%$ em STAI 2 & $59,8 \%$ & $40,2 \%$ & $100,0 \%$ & & \\
\hline & & $\%$ em SOC & $100,0 \%$ & $100,0 \%$ & $100,0 \%$ & & \\
\hline & & & BDI-II & & & & \\
\hline & & & $\begin{array}{l}\text { Depressão } \\
\text { mínima }\end{array}$ & $\begin{array}{l}\text { Depressão } \\
\text { leve }\end{array}$ & $\begin{array}{l}\text { Depressão } \\
\text { moderada }\end{array}$ & TOTAL & p-valor \\
\hline \multirow[t]{9}{*}{ STAI 2} & Baixo & Contagem & 21 & 0 & 0 & 21 & \\
\hline & & $\%$ em STAI 2 & $100 \%$ & $0,0 \%$ & $0,0 \%$ & $100,0 \%$ & \\
\hline & & $\%$ em BDI-II & $23,3 \%$ & $0,0 \%$ & $0,0 \%$ & $20,6 \%$ & \\
\hline & Moderado & Contagem & 59 & 4 & 0 & 63 & $<0,001 * *$ \\
\hline & & $\%$ em STAI 2 & $93,7 \%$ & $6,3 \%$ & $0,0 \%$ & $100,0 \%$ & \\
\hline & & $\%$ em BDI-II & $65,6 \%$ & $50.0 \%$ & $0,0 \%$ & $61,8 \%$ & \\
\hline & Alto & Contagem & 10 & 4 & 4 & 18 & \\
\hline & & $\%$ em STAI 2 & $55,6 \%$ & $22,2 \%$ & $22,2 \%$ & $100 \%$ & \\
\hline & & $\%$ em BDI-II & $11,1 \%$ & $50,0 \%$ & $100,0 \%$ & $17,6 \%$ & \\
\hline \multirow[t]{3}{*}{ TOTAL } & & Contagem & 90 & 8 & 4 & 102 & \\
\hline & & $\%$ em STAI 2 & $88,2 \%$ & $7,8 \%$ & $3,9 \%$ & $100,0 \%$ & \\
\hline & & $\%$ em BDI-II & $100 \%$ & $100 \%$ & $100,0 \%$ & $100.0 \%$ & \\
\hline
\end{tabular}

(*) Teste Qui-Quadrado de Pearson (**) Teste Exato de Fischer Fonte: Autores.

\section{Segunda coleta de dados}

A segunda coleta de dados foi realizada no final do primeiro ano de curso e contou com 98 (79\%) dos participantes dos 123 iniciais (Tabela 3).

O questionário de Senso de coerência que mediu o grau de resiliência dos estudantes (SOC) apresentou baixa resiliência para a maioria dos participantes $(51,2 \%)$ na primeira coleta de dados e alta resiliência para $55,1 \%$ dos participantes na segunda coleta de dados. O instrumento utilizado para calcular a ansiedade, o STAI, apresentou a maioria com moderado nível de ansiedade-traço $(51,2 \%)$ e baixa ansiedade-estado em $46,3 \%$ dos participantes da pesquisa na primeira coleta de 
dados. Na segunda coleta de dados os pós-graduandos reportaram níveis de ansiedade-estado e traço ambos moderados (46\% e 45,9\%), embora houve uma diminuição dos estudantes que reportaram baixa ansiedade-estado (de 46,3\% para 17,3\%), este resultado foi estatisticamente significativo $(\mathrm{p}<0,001)$. Com relação a aferição da depressão, o BDI-II apresentou participantes com depressão mínima $(90,2 \%)$, leve $(6,5 \%)$ e moderada $(3,3 \%)$ e nenhum participante apresentou depressão severa na primeira coleta de dados. Na segunda coleta de dados, 2 alunos apresentaram depressão severa, 20,4\% depressão leve, $5,1 \%$ apresentaram depressão moderada e 72,5\% com mínima depressão.

Tabela 3: Distribuição dos valores dos índices SOC, STAI-1 (ansiedade-estado), STAI-2 (ansiedade-traço) e BDI-II entre estudantes de pós-graduação UPE e UFPB na primeira e segunda coleta de dados.

\begin{tabular}{|c|c|c|c|}
\hline \multirow[b]{2}{*}{ Variáveis } & \multicolumn{2}{|c|}{ Momentos } & \multirow[b]{2}{*}{ p-valor } \\
\hline & $\begin{array}{c}1^{\mathbf{0}} \text { Momento } \\
\text { n (\%) }\end{array}$ & $\begin{array}{c}2^{\mathbf{o}} \text { Momento } \\
\text { n (\%) }\end{array}$ & \\
\hline \multicolumn{4}{|l|}{ SOC } \\
\hline Alto (> 46) & $60(48,8)$ & $54(55,1)$ & $0,350 *$ \\
\hline Baixo (até 46) & $63(51,2)$ & $44(44,9)$ & \\
\hline \multicolumn{4}{|l|}{ STAI 1} \\
\hline Alto/sério: $\geq 50$ pontos & $18(14,6)$ & $36(36,7)$ & $<0,001 *$ \\
\hline Moderado: 35 a 49 pontos & $48(39,0)$ & $45(45,9)$ & \\
\hline Baixo: 20 a 34 pontos & $57(46,3)$ & $17(17,3)$ & \\
\hline \multicolumn{4}{|l|}{ STAI 2} \\
\hline Alto/sério: $\geq 50$ pontos & $18(14,6)$ & $27(27,6)$ & $0,055 *$ \\
\hline Moderado: 35 a 49 pontos & $63(51,2)$ & $45(45,9)$ & \\
\hline Baixo: 20 a 34 pontos & $42(34,1)$ & $26(26,5)$ & \\
\hline \multicolumn{4}{|l|}{ BDI II } \\
\hline Depressão severa: 29-63 & $0(0,0)$ & $2(2,0)$ & $0,002 * *$ \\
\hline Depressão moderada: 20-28 & $4(3,3)$ & $5(5,1)$ & \\
\hline Depressão leve: 14-19 & $8(6,5)$ & $20(20,4)$ & \\
\hline Depressão nenhuma/ mínima: 0-13 & $111(90,2)$ & $71(72,4)$ & \\
\hline
\end{tabular}

(*) Teste Qui-Quadrado (**) Teste Exato de Fisher Fonte: Autores.

A diferença de valores entre a primeira e segunda coleta referente a STAI-1 teve significância estatística $(\mathrm{p}<0,001)$ demonstrando que a ansiedade alta/séria aumentou significativamente na segunda coleta de dados: $14,6 \%$ dos participantes tinham ansiedade alta/ séria na primeira coleta de dados tendo aumentado para 36,7\% da amostra na segunda coleta.

\section{Discussão}

O crescimento da prevalência e severidade das desordens mentais entre estudantes universitários pós-graduandos tem sido de interesse da comunidade acadêmica mundial e dos órgãos de saúde. No presente estudo, onde foi avaliado o perfil social, estudantil e econômico dos estudantes de pós-graduação de duas Universidades nordestinas, houve maior prevalência do sexo feminino, igualmente a estudos nacionais e internacionais (Levecque et al., 2017; Faro, 2013; Monrouxe et al., 2017; 
Racic et al., 2017; Kamat et al., 2015; Nagy et al, 2019), a idade dos participantes variou de 21 a 69 anos, a maioria entre 20 e 30 anos, semelhante a estudos (Costa \& Nebel, 2018; Almalik et al., 2018). No presente estudo a maioria dos estudantes não tinha filhos como encontrado em outros trabalhos (Costa \& Nebel, 2018; Faro, 2013; Levecque et al., 2017; Macauley et al., 2018) e era solteiro (Costa \& Nebel, 2018).

Com relação ao gênero, os achados de nosso estudo corroboram os resultados de Almalik et al. (2018) no qual encontrou altos níveis de ansiedade em mulheres mais do que em homens (78.3\% comparado com 21,7\%) e em estudantes pósgraduandos mais jovens (76,7\% comparado com 23.3\%). Foi verificada a ansiedade e a depressão em 325 pós-graduandos na China (Liu et al., 2019) onde a maioria das mulheres (42,3\%) apresentou depressão leve e a maioria dos participantes da pesquisa era casado ou vivia com o parceiro (Costa \& Nebel, 2018, Faro 2013). No estudo de Levecque et al. (2018) as mulheres tinham 34\% maior risco de apresentarem sintomas de sofrimento mental (depressão). Na Universidade do Arizona (Smith \& Brooks, 2015) foi avaliado o impacto da pós-graduação na saúde de estudantes com o objetivo de criar intervenções benéficas no ambiente estudantil e os autores verificaram que 55\% e 57\% dos transgêneros apresentaram ansiedade e depressão, comparados com $43 \%$ e $41 \%$ das mulheres e em $34 \%$ e $35 \%$ dos homens, respectivamente. Liu et al., (2019) relataram que 42,3\% das mulheres tinham depressão mínima semelhante aos casados (43\%), os respondentes que não tinham filhos se mostraram em sua maioria sem depressão $(40,5 \%)$ e os que tinham ao menos um filho, por sua vez, apresentaram depressão mínima (46,2\%).

Mazurek Melnyk et al. (2020) que conduziram um estudo descritivo com estudantes pós-graduandos de sete diferentes cursos de uma universidade do meio oeste dos Estados Unidos, demonstraram que mais da metade da amostra era de mulheres $(64,5 \%)$ e $50,8 \%$ destas apresentaram depressão elevada, $41 \%$ dos estudantes relataram sintomas indicativos de elevada depressão e 27,8\% elevada ansiedade. No trabalho de Nagy et al. (2019) em que foram estudadas as variáveis socioeconômicas, demográficas, acadêmicas e de saúde mental em 69 pós-graduandos de biomedicina de uma instituição do Sudoeste dos Estados Unidos, a média de idade era de 26,5 anos, sua maioria mulheres (60,9\%) igualmente ao nosso estudo (65\%), porém, a maioria em relacionamento estável, mas não casado (44,9\%). Aproximadamente metade dessa amostra $(52,2 \%)$ apresentou diagnóstico de pelo menos um transtorno psiquiátrico e os mais prevalentes nesse estudo foram transtornos de ansiedade (31,9\%); em análise comparativa com a população total dos EUA os alunos doutorandos em biomedicina desse estudo (Nagy et al., 2019) apresentaram maior prevalência de distúrbios psiquiátricos (49,3\%) do que a população total dos EUA $(26,2 \%)$.

Macauley et al (2018) em seu estudo descrevem a prevalência e determinam os preditores significativos de ansiedade nos alunos de profissões de assistência à saúde da Universidade de Husson (USA), onde 51\% das mulheres e 37,5\% dos homens apresentaram ansiedade moderada a alta. Evans et al. (2018) avaliaram ansiedade e depressão em 2,279 pósgraduandos em 26 países onde $41 \%$ dos pós-graduandos apresentaram ansiedade moderada a severa e 39\% depressão moderada a severa. Racic et al. (2015) avaliaram a ansiedade e depressão em estudantes de medicina, odontologia, enfermagem e fonoaudiologia em uma faculdade na Bósnia e Herzegovina e identificaram que a maioria da sua amostra era de mulheres $(69,2 \%)$ que relataram depressão mais frequentemente em comparação com os estudantes do sexo masculino. No presente estudo identificamos que na primeira coleta de dados os participantes em sua maioria apresentaram depressão mínima $(90,2 \%)$ e nenhum aluno apresentou depressão severa, na segunda coleta de dados $71 \%$ apresentaram depressão mínima e $2 \%$ dos alunos apresentaram depressão severa.

Outro ponto importante na avaliação da saúde mental de estudantes de pós-graduação se refere a situação econômica. Nosso estudo observou que os pós-graduandos em sua maioria fazem parte de famílias que tem uma renda média de 3 saláriosmínimos (R \$ 3.300,00 ou 584,07 dólares mensais). No estudo de Liu et al (2019) os participantes que tinham renda familiar menor do que $3000 \mathrm{CN} ¥(\mathrm{R} \$ 2.563,53)$ per capta apresentaram depressão leve, igualmente aos que tinham renda maior do que 
$5.000 \mathrm{CN} ¥(\mathrm{R} \$ 4.272,55)$ per capta $(46,8 \%)$; a maioria dos participantes $(56,6 \%)$ tinha a renda mensal menor que $3000 \mathrm{CN} ¥$ ( $\mathrm{R} \$ 2.563,53)$. Costa \& Nebel (2018) relataram em seu estudo que $76 \%$ dos estudantes recebiam bolsa, sendo o gasto mensal com a pós-graduação de aproximadamente 700 reais (ou 125,89 dólares).

No presente estudo a maioria dos pós-graduandos citou trabalhar ao mesmo tempo em que cursa o mestrado (58,5\%), igualmente ao estudo de Smith \& Brooks (2015), onde 63\% da sua amostra de estudantes relatou trabalhar e ser esse um fator contribuinte para a ansiedade e stress acadêmico maior do que a média, igualmente educação (72\%) e questões financeiras (55\%). No estudo de Hoying et al. (2020) foi avaliado a prevalência da depressão, ansiedade, stress, saúde física e estilos de vida em estudantes pós-graduandos de saúde onde 42,6\% relatou não trabalhar. Costa \& Nebel (2018) relataram que 71\% dos estudantes entrevistados afirmaram não exercer outra atividade profissional além da pós-graduação. Em pesquisa realizada em 2018, com participação de 637 estudantes de pós-graduação da Universidade de Brasília (UnB) foi afirmado que 52\% dos respondentes não tinham trabalho além da pós-graduação e 52\% dos participantes recebiam bolsa (Oliveira \& Conceição, 2018).

Estudantes pós-graduandos sofrem com a modificação no padrão de sono devido a necessidade de estender as horas de estudo interferindo assim nas horas anteriormente destinadas para o sono. Frequentemente, esses fatores resultam em problemas de longo prazo como o dia sonolento, instabilidade emocional e exaustão mental, dificultando o processo de ensinoaprendizagem. No presente estudo, $82,1 \%$ dos participantes relataram não ter problemas para dormir. No estudo de Smith \& Brooks (2016), os estudantes de mestrado mostraram uma queda na classificação subjetiva de padrão de sono desde o início dos estudos de pós-graduação, a maioria (74\%) dos estudantes de mestrado relatou que dormem o suficiente em 2 a 5 noites por semana e $24 \%$ disseram que o sono era pior desde o início dos estudos de pós-graduação. Costa \& Nebel (2018) verificaram que $31 \%$ dos pesquisados sofriam com insônia e $62 \%$ dormiam diariamente 7 horas ou mais. No estudo de Hoyig et al. (2020), 38,1\% dos participantes tinham menos de 7 horas de sono por dia. O estudo de Komarov, Ko, \& Jung (2020) demonstrou correlação positiva de fadiga percebida com estresse, sonolência diurna e negativa com atenção ao despertar, qualidade do sono autoavaliada e duração do sono.

Durante a pós-graduação existem desafios para que se continue um equilíbrio na vida estudantil no que diz respeito a práticas de atividades saudáveis como exercícios físicos regulares, considerando os prazos, avaliações, apresentações, ritmos de trabalho, que muitas vezes não permitem a priorização da realização desse autocuidado. No presente estudo, $62,6 \%$ dos participantes relataram fazer atividade física, destes 26,8\% faziam 3 vezes por semana e 9,8\% 5 vezes na semana. No estudo de Hoying et al. (2020) 57,4\% não atendeu à quantidade recomendada de atividade física (ou seja, 30 min ou mais pelo menos cinco vezes por semana). No estudo de Mazurek Melnyk et al. (2020) onde foram avaliados 93 pós-graduandos de cursos de saúde de uma grande universidade no meio oeste dos EUA, 44\% exercitavam 30 minutos por dia 5 vezes na semana embora quase $40 \%$ dos estudantes tinham um critério de sobrepeso ou obesidade de acordo com o IMC. No estudo de Smith \& Brooks (2016) a maioria dos pós-graduandos relatou que sua rotina de exercícios ficou pior (62\%) ou não mudou (17\%) desde o início dos estudos de pós-graduação/profissional.

Foram encontrados na primeira coleta de dados deste estudo níveis de baixa ansiedade: estado (46,3\%) e traço (34,1\%), níveis moderados de ansiedade estado (39\%) e traço (51,2\%) e alta ansiedade ambos no estado e traço (14,6\%). Na segunda coleta houve uma diminuição dos alunos que reportaram baixa ansiedade estado (de 46,3\% para 17,3\%) (p<0,001). Durante a segunda coleta de dados (final do ano letivo) ocorreu uma diferença estatisticamente significativa entre os valores referentes a ansiedade medida pelo STAI-estado (aumento de 14,6\% alta ansiedade medida no primeiro momento para 36,7\% no segundo momento) e a depressão medida pelo BDI-II onde 2 participantes relataram depressão severa. A depressão leve aumentou de 6,5\% para 20,4\% demonstrando uma diferença nos estados do início e meio do curso, sugerindo aumento de depressão e ansiedade nos momentos de apresentação da qualificação. No estudo de Almalink et al., (2018) foi demonstrado 
que alunos de pós-graduação em seu último ano (sênior) eram mais propensos a ficarem ansiosos durante o diagnóstico clínico e as investigações do que os alunos de pós-graduação do primeiro ano (juniores).

Em relação ao senso de coerência, o presente estudo apresentou um aumento de valor dos participantes da primeira coleta $(48,8 \%)$ para o segundo momento $(55,1 \%)$. Ito et al. (2018) realizou um estudo follow-up em estudantes japoneses onde na primeira coleta de dados, dos 1.738 respondentes iniciais, 169 residentes testaram positivo para sintomas depressivos, na segunda coleta de dados 187 residentes (19,5\%) reportaram sintomas depressivos, dos quais 33,3\% apresentaram um baixo SOC e 11,4\% alto. Ainda nesse estudo, o escore SOC estava significativamente relacionado a depressão entre médicos residentes 2 anos após o início de treinamento clínico (Ito et al., 2018). Na avaliação da resiliência, Garista et al. (2019), em seu estudo apresentou uma interpretação da aprendizagem acadêmica com fundamentos da teoria salutogênica em um curso de pós-graduação em Promoção e Educação para a Saúde, da Universidade de Perugia, Itália no qual estudantes pós-graduandos desenharam como se sentiam no início e final do curso e explanaram sobre os desenhos, associando a reflexões sobre o que era a salutogênese e o que podiam considerar uma aplicação clara para o processo de aprendizagem. Nesse estudo de Garista et al. (2019) os autores relataram que os alunos reportaram realizar uma reflexão através dos desenhos, visualizando aspectos da aprendizagem e multiplicando as possibilidades de comunicação em tarefas e situações. Wahab et al. (2017) guiados pela teoria do Senso de Coerência, exploraram os relatos de 9 enfermeiras pós-graduandas utilizando fotografias digitais tiradas pelas participantes que descreveram e relataram o que é ser resiliente e a facilidade/impedimento da construção dessa resiliência em suas vidas. Nesse estudo, as participantes da pesquisa responderam que apesar do ambiente de trabalho ser estressante e dos obstáculos encontrados, a resiliência é construída sobre a compreensibilidade, significância e gestão de qualquer situação que elas encaram em sua vida pessoal, profissional e espiritual (Wahab et al., 2017).

\section{Conclusão}

Houve um aumento do senso de coerência, manifestação da depressão severa, ansiedade traço no nível moderado e o aumento da ansiedade estado entre os alunos pós-graduandos pesquisados durante o desenvolvimento do primeiro ano de pósgraduação.

\section{Agradecimentos}

O presente trabalho foi realizado com apoio da Coordenação de Aperfeiçoamento de Pessoal de Nível Superior Brasil (CAPES) - Código de Financiamento 001

\section{Referências}

Almalik, M., Alnowaiser, A., El Meligy, O., Sallam, J., \& Balkheyour, Y. (2018). Clinical Anxiety among Saudi Postgraduate Pediatric Dentistry Students in Jeddah City. International journal of dentistry, 2018, 5863869. 10.1155/2018/5863869

American Psychiatric Association - APA. (2014). Manual diagnóstico e estatístico de transtornos mentais: DSM-5 (5a ed.), Artmed.

Antonovsky A. (1993). The structure and properties of the sense of coherence scale. Social science \& medicine (1982), 36(6), 725-733. 10.1016/02779536(93)90033-z.

Beck, A. T., Ward, C. H., Mendelson, M., Mock, J., \& Erbaugh, J. (1961). An inventory for measuring depression. Archives of general psychiatry, 4, 561-571. 10.1001/archpsyc.1961.01710120031004

Costa, E. G., \& Nebel, L. (2018) O quanto vale a dor? Estudo sobre a saúde mental de estudantes de pós-graduação no Brasil. Polis, 50, 207-227 http://journals.openedition.org/polis/15816

Costa G. (set, 2019). Capes anuncia corte de 5613 bolsas de pós-graduação para este ano. Agencia Brasil, Brasília, DF. https://agenciabrasil.ebc.com.br/educacao/noticia/2019-09/capes-anuncia-corte-de-5613-bolsas-de-pos-graduacao-para-este-ano.shtml 
Cirani, C. B., Silva, Campanario, M. A. \& Silva, H. H. M. (2015). A evolução do ensino da pós-graduação senso estrito no Brasil: análise exploratória e proposições para pesquisa. Avaliação: Revista da Avaliação da Educação Superior (Campinas), 20(1), 163-187. 10.590/S1414-40772015000500011

Garcia da Costa, E., \& Nebel, L. (2018). O quanto vale a dor? Estudo sobre a saúde mental de estudantes de pós-graduação no Brasil. Polis (Santiago), 17(50), 207-227. 10.4067/S0718-65682018000200207

Evans, T. M., Bira, L., Gastelum, J. B., Weiss, L. T., \& Vanderford, N. L. (2018). Evidence for a mental health crisis in graduate education. Nature biotechnology, 36(3), 282-284. 10.1038/nbt.4089

Faro, A. (2013). Estresse e estressores na pós-graduação: estudo com mestrandos e doutorandos no Brasil. Psicologia: Teoria e Pesquisa, 29(1), 51-60. $10.1590 / \mathrm{S} 0102-37722013000100007$

Freire, M. C., Sheiham, A., \& Hardy, R. (2001). Adolescents' sense of coherence, oral health status, and oral health-related behaviours. Community dentistry and oral epidemiology, 29(3), 204-212. 10.1034/j.1600-0528.2001.290306.X

Garista, P., Pocetta, G., \& Lindström, B. (2019). Picturing academic learning: salutogenic and health promoting perspectives on drawings. Health promotion international, 34(4), 859-868. 10.1093/heapro/day027

Greimel, E., Kato, Y., Müller-Gartner, M., Salchinger, B., Roth, R., \& Freidl, W. (2016). Internal and External Resources as Determinants of Health and Quality of Life. PloS one, 11(5), e0153232. 10.1371/journal.pone.0153232

Hoying, J., Melnyk, B. M., Hutson, E., \& Tan, A. (2020). Prevalence and Correlates of Depression, Anxiety, Stress, Healthy Beliefs, and Lifestyle Behaviors in First-Year Graduate Health Sciences Students. Worldviews on evidence-based nursing, 17(1), 49-59. 10.1111/wvn.12415

Iriart, Jorge Alberto Bernstein, \& Trad, Leny Alves Bomfim. (2020). Perfil da produção bibliográfica em Ciências Sociais e Humanas em Saúde e a percepção de pesquisadores: avanços, limites e desafios. Cadernos de Saúde Pública, 36(5), e00085019. 10.1590/0102-311x00085019

Ito, M., Seo, E., Maeno, T., Ogawa, R., \& Maeno, T. (2018). Relationship Between Depression and Stress Coping Ability Among Residents in Japan: A TwoYear Longitudinal Study. Journal of clinical medicine research, 10(9), 715-721. 10.14740/jocmr3512w

Jeong, Y. J., \& Koh, C. K. (2021). Female nursing graduate students' stress and health: the mediating effects of sense of coherence and social support. BMC nursing, 20(1), 40. 10.1186/s12912-021-00562-x

Kamat, C. A., Todakar, M., Rangalakshmi, S., \& Pawan (2015). Awareness about scope of anaesthesiology, attitudes towards the speciality and stress levels amongst postgraduate students in anaesthesiology: A cross-sectional study. Indian journal of anaesthesia, 59(2), 110-117. 10.4103/0019-5049.151375

Klawonn, A., Kernan, D., \& Lynskey, J. (2019). A 5-Week Seminar on the Biopsychosocial-Spiritual Model of Self-Care Improves Anxiety, SelfCompassion, Mindfulness, Depression, and Stress in Graduate Healthcare Students. International journal of yoga therapy, 29(1), 81-89. 10.17761/D-18-201900026

Komarov, O., Ko, L. W., \& Jung, T. P. (2020). Associations Among Emotional State, Sleep Quality, and Resting-State EEG Spectra: A Longitudinal Study in Graduate Students. IEEE transactions on neural systems and rehabilitation engineering: a publication of the IEEE Engineering in Medicine and Biology Society, 28(4), 795-804. 10.1109/TNSRE.2020.2972812

Levecque, K., Anssel, F., De Beuckelaer, A., Van der Heyden, J., Gisle, L. (2017). Work organization and mental health problems in PhD students. Res Policy,46, 868-879. 10.1016/j.respol.2017.02.008

Lindmark, U., Ahlstrand, I., Ekman, A., Berg, L., Hedén, L., Källstrand, J., Larsson, M., Nunstedt, H., Oxelmark, L., Pennbrant, S., Sundler, A., Larsson, I., \& Impact within the Swedish framework for "Health Research in Collaboration" (2020). Health-promoting factors in higher education for a sustainable working life - protocol for a multicenter longitudinal study. BMC public health, 20(1), 233. 10.1186/s12889-020-8181-3

Lindström, B., \& Eriksson, M. (2011). From health education to healthy learning: implementing salutogenesis in educational science. Scandinavian journal of public health, 39(6 Suppl), 85-92. 10.1177/1403494810393560

Liu, C., Wang, L., Qi, R., Wang, W., Jia, S., Shang, D., Shao, Y., Yu, M., Zhu, X., Yan, S., Chang, Q., \& Zhao, Y. (2019). Prevalence and associated factors of depression and anxiety among doctoral students: the mediating effect of mentoring relationships on the association between research self-efficacy and depression/anxiety. Psychology research and behavior management, 12, 195-208. 10.2147/PRBM.S195131

Mazurek Melnyk, B., Slevin, C., Militello, L., Hoying, J., Teall, A., \& McGovern, C. (2016). Physical health, lifestyle beliefs and behaviors, and mental health of entering graduate health professional students: Evidence to support screening and early intervention. Journal of the American Association of Nurse Practitioners, 28(4), 204-211. 10.1002/2327-6924.12350

Macauley K, Plummer L, Bemis C, Brock G, Larson C, Spangler J. (2018). Prevalence and predictors of anxiety in health care professions students. Health Professions Education, 4, 176-185. 10.1016/j.hpe.2018.01.001

Mirza, W., Mirza, A. M., Saleem, M. S., Chacko, P. P., Ali, M., Tarar, M. N., Babar, A., Freiwald, J., \& Talitskiy, K. (2018). Well-being Assessment of Medical Professionals in Progressive Levels of Training: Derived from the WHO-5 Well-being Index. Cureus, 10(12), e3790. 10.7759/cureus.3790

Monrouxe, L. V., Bullock, A., Tseng, H. M., \& Wells, S. E. (2017). Association of professional identity, gender, team understanding, anxiety and workplace learning alignment with burnout in junior doctors: a longitudinal cohort study. BMJ open, 7(12), e017942. 10.1136/bmjopen-2017-017942

Nagy, G. A., Fang, C. M., Hish, A. J., Kelly, L., Nicchitta, C. V., Dzirasa, K., \& Rosenthal, M. Z. (2019). Burnout and Mental Health Problems in Biomedical Doctoral Students. CBE life sciences education, 18(2), ar27. 10.1187/cbe.18-09-0198

Oliveira, H. A. L., Conceição, M. I. G. (2018). Sofrimento mental de pós-graduandos na UNB: Levantamento e propostas de solução. Anais do 6º Congresso Brasileiro de saúde mental: Agir e transformar: pessoas, afetos e conexões, Brasília - DF Anais (on-line). Brasília: http://www.congresso2018.abrasme.org.br/site/anaiscomplementares2?AREA=6\#php2go_top 
Research, Society and Development, v. 10, n. 7, e28610716473, 2021

(CC BY 4.0) | ISSN 2525-3409 | DOI: http://dx.doi.org/10.33448/rsd-v10i7.16473

Puthran, R., Zhang, M. W., Tam, W. W., \& Ho, R. C. (2016). Prevalence of depression amongst medical students: a meta-analysis. Medical education, 50(4), 456-468. 10.1111/medu.12962

Racic, M., Todorovic, R., Ivkovic, N., Masic, S., Joksimovic, B., \& Kulic, M. (2017). Self- Perceived Stress in Relation to Anxiety, Depression and Healthrelated Quality of Life among Health Professions Students: A Cross-sectional Study from Bosnia and Herzegovina. Zdravstveno varstvo, 56(4), 251-259. 10.1515/sjph-2017-0034

Smith, E., \& Brooks, Z. (University of Arizona.). (2015). Graduate student mental health 2015. National Association of graduate-professional student and the graduate professional student council (GPSC). http://nagps.org/wordpress/wp-content/uploads/2015/06/NAGPS_Institute_mental_hea 1th_survey_report_2015.pdf

Spielberger, C. D., Gorssuch, R. L., Lushene, P. R., Vagg, P. R., \& Jacobs, G. A. (1983). Manual for the State-Trait Anxiety Inventory. Consulting Psychologists Press. https://www.researchgate.net/publication/235361542_Manual_for_the_State-Trait_Anxiety_Inventory_Form_Y1_-_Y2

UPE/Pós-graduação, Pesquisa e Inovação (2021). http://www.upe.br/pos-graduacao-e-pesquisa.html

UFPB/PRPG/Pró-reitoria de pós-graduaçao (2021). http://www.prpg.ufpb.br/prpg/contents/paginas/programas-de-pos-graduacao/lista-de-cursos-de-posgraduacao

Wahab, S., Mordiffi, S. Z., Ang, E., \& Lopez, V. (2017). Light at the end of the tunnel: New graduate nurses' accounts of resilience: A qualitative study using Photovoice. Nurse education today, 52, 43-49. 10.1016/j.nedt.2017.02.007

Wanderley, M. B. (2017). Dilemmas and challenges posed to Graduate Studies in Brazil. Revista Katálysis, 20(2), 142-144. 10.1590/1982$02592017 \mathrm{v} 20 \mathrm{n} 2 \mathrm{p} 142$

Woolston C. (2019). PhDs: the tortuous truth. Nature, 575(7782), 403-406. 10.1038/d41586-019-03459-7

World Health Organization. (2017). Depression and other common mental disorders: global health estimates. World Health Organization. Geneva: WHO 2017 24 p. https://apps.who.int/iris/handle/10665/254610

Zhang, Y., Liu, R., Li, G., Mao, S., \& Yuan, Y. (2015). The reliability and validity of a Chinese-version Short Health Anxiety Inventory: an investigation of university students. Neuropsychiatric disease and treatment, 11, 1739-1747. 10.2147/NDT.S83501

World Health Organization. (2020a). Mental health and psychosocial considerations during the COVID-19 outbreak Geneva: Author. https://www.who.int/docs/default-source/coronaviruse/mental-health-considerations.pdf 